\title{
PATIENTS WITH SYSTEMIC SCLEROSIS ASSOCIATING OTHER AUTOIMMUNE DISEASES HAVE A MILDER DISEASE COURSE
}

\author{
Alina Soare, Simona Pintilie, Ana Maria Gherghe, Alexandra Radu, Rucsandra Dobrota, \\ Mariana Sasu, Mihai Bojinca, Victor Stoica, Carina Mihai \\ EUSTAR centre 100, Internal Medicine and Rheumatology Department, \\ Dr. I. Cantacuzino Hospital, Carol Davila University of Medicine and Pharmacy, Bucharest, Romania
}

Abstract
Background and objectives. Systemic sclerosis (SSc) is a multisystem connective tissue disease (CTD), being
one of the most heterogeneous diseases of the spectrum of CTDs. It may associate other autoimmune diseases
(AIDs), therefore in this study we aimed to evaluate the prevalence of other AIDs in a cohort of patients with SSc
and to evaluate their prognosis in comparison to patients with SSc without this association.
Patients and methods. We performed a retrospective study in patients with SSc satisfying the ACR 1980 or the
ACR/EULAR 2013 criteria, who were evaluated between January 2005 and May 2014 in our SSc center. These
patients were investigated according to the EUSTAR recommendations, including modified Rodnan Skin Score
(mRSS), lung function tests (LFT), echocardiography etc. As a control group for evaluating the prevalence of the
AIDs we used all patients with Rheumatoid Arthritis (RA) visiting our clinic between January-December 2005.
Data of all RA patients were collected from the electronic database of the hospital. All AIDs mentioned in the
discharge diagnoses were recorded. Between-group comparisons were made with the chi-square test for nominal
variables and with the independent-sample t-test for numeric variables.
Results. 144 patients with SSc were included: $88.8 \%$ females, $66.6 \%$ with the limited cutaneous subset of dis-
ease (IcSSc), mean age $53.9 \pm 12.8$ years, mean disease duration at study enrollment $5.1 \pm 10.5$ years and a
mean follow-up time of $3.7 \pm 2.6$ years. Prevalence of the AID in the SSc cohort was $19.4 \%$, patients with IcSSc
being more prone to associate other AID ( $p=0.001)$. We noted a tendency towards less interstitial lung disease
(p=0.056) and less digital ulcers ( $p=0.081$ ) in patients with SSc and AIDs. Comparing skin involvement for each
year of follow-up in patients with SSc with and without and AIDs we observed that the first tended to have lower
and more stable values of the mRSS.
Conclusion. Patients with SSc and AIDs tend to have a better outcome than the ones without this association.
mRSS remains stable across the years in patients with SSc and AID, with lower values than the SSc patients.
Keywords: systemic sclerosis, autoimmune disease, polyautoimmunity

Systemic sclerosis (SSc) is a chronic, multisystem disorder associated with the highest mortality among the connective tissue diseases (CTDs) (1). Being not only the prototype of fibrotic diseases but also an autoimmune and vascular disease, SSc is a heterogeneous condition with variable clinical presentations and course, frequently associating other autoimmune conditions - which makes the management of these patients more difficult.

Despite all efforts to understand the pathogenesis of SSc, some pieces of the puzzle are still missing. In the last years, studies brought into light evidence that SSc shares a genetic background with other au- toimmune diseases (AID) and certain genes can predispose to multiple AIDs (2). It has long been known that SSc can be associated with other autoimmune rheumatologic diseases ("overlap syndromes") and also with other autoimmune conditions, but the prevalence of these associations in SSc is still a matter of debate.

In this study we included under the name of AIDs any other connective tissue diseases except SSc, and also antiphospholipid antibody syndrome, rheumatoid arthritis, autoimmune thyroiditis, autoimmune hepatitis, primary biliary cirrhosis and type I diabetes mellitus (3). A disease is regarded as an autoim-

Correspondence address:

Alina Soare, MD, PhD student, Carol Davila University of Medicine and Pharmacy, 37 Dionisie Lupu St., Bucharest, Romania

E-mail: alinasoare@gmx.com 
mune disease when it meets the Witebsky's postulates (direct evidence from transfer of pathogenic antibody or pathogenic $\mathrm{T}$ cells, indirect evidence based on reproduction of the immune disease in experimental animals and circumstantial evidence from clinical clues) (4).

The aim of our study is to determine the prevalence of AIDs in the cohort of patients with SSc of the EUSTAR center 100 and to compare the outcome of these patients with the ones without an associated AID.

\section{PATIENTS AND METHODS}

We performed a retrospective study by reviewing the charts of all SSc patients, diagnosed according to ACR 1980 criteria or the ACR/EULAR 2013, who were evaluated between January 2005 and May 2014 in the EUSTAR center 100 (Dr. Ion Cantacuzino Clinical Hospital, Bucharest, Romania), with regard to the association of another AID. Patients were investigated yearly according to the EUSTAR recommendations, including the modified Rodnan Skin Score (mRSS), blood tests, lung function tests (LFT), echocardiography, chest x-Ray etc. All SSc patients have given written informed consent for participation in the EUSTAR database and in any observational study conducted on EUSTAR data. We registered any diagnosis of one of the following diseases: Rheumatoid Arthritis (RA), Systemic Lupus Erythematosus (SLE), Sjögren's Syndrome (SS), Dermatomyositis (DM), Polymyositis (PM), Autoimmune Thyroiditis (AIT), Primary Biliary Cirrhosis (PBC), Type 1 Diabetes Mellitus (Type 1 DM), antiphospholipid syndrome (APLS). Diagnosis of the associated disease was made according to the expert opinion. As a control group for evaluating the prevalence of AIDs we used all patients with Rheumatoid Arthritis (RA) visiting during the year 2005 the rheumatology clinic of the same hospital, followed-up until May 2014. Patients diagnosed with both SSc and RA were analyzed in the SSc group. We compared the patients with SSc to the ones with RA regarding the prevalence of AIDs. We also compared the SSc patients associating any AIDs to SSc patients without AIDs in regard to mortality, pulmonary function tests, and vascular impairment, aiming to determinate the prognosis of these patients. Data on AIDs of SSc patients and all data of RA patients were collected from the electronic database of the hospital. The study was approved by the local ethics committee. The statistical analysis was performed using the IBM SPSS 20.0 software. Between-group comparisons were made with the chi-square test for nominal variables and with the independent-sample t-test for numeric variables. A p-value below 0.05 was considered statistically significant.

\section{RESULTS}

We included 144 patients with SSc, among which $88.8 \%$ females, and $66.6 \%$ with limited cutaneous $\mathrm{SSc}(\mathrm{lcSSc})$. The control group included 511 patients with RA. Demographic data of the SSc and RA patients are presented in Table 1.

TABLE 1. Demographic data of the patients with SSC and the control patients with $R A$

\begin{tabular}{|l|c|c|}
\hline & $\begin{array}{c}\text { SSc patients } \\
\text { ( } \mathbf{n = 1 4 4 )}\end{array}$ & $\begin{array}{c}\text { RA } \\
\text { (n = 511) }\end{array}$ \\
\hline $\begin{array}{l}\text { Age at inclusion, years } \\
\text { (mean } \pm \text { SD) }\end{array}$ & $54.3 \pm 12.3$ & $61,6 \pm 13.2$ \\
\hline $\begin{array}{l}\text { Age at diagnosis, years } \\
\text { (mean } \pm \text { SD) }\end{array}$ & $47.76 \pm 12.91$ & $49 . \pm 14.9$ \\
\hline $\begin{array}{l}\text { Disease duration, years } \\
\text { (mean } \pm \text { SD) }\end{array}$ & $6.5 \pm 6.4$ & $12.6 \pm 9.3$ \\
\hline Female gender & $128(88.9 \%)$ & $419(82.0 \%)$ \\
\hline Deceased before May 2014 & $14(9.7 \%)$ & $6(1.2 \%)$ \\
\hline
\end{tabular}

\section{Prevalence of AIDs in the SSc cohort}

The overall prevalence of AIDs inSSc patients was $19.4 \%$, with RA being the most frequent association, followed by SLE (5.6\%) and AIT (4.9\%) (Table 2). In the RA group the overall prevalence of AIDs was only $4.7 \%$, SS (2.3\%) and AIT (1.8\%) being the most frequent associations (Table 2). We also

TABLE 2. Prevalence of associated autoimmune diseases (AIDs) in SSc patients and in the RA control cohort

\begin{tabular}{|l|c|c|c|}
\hline & $\begin{array}{c}\text { SSc patients } \\
(n=144)\end{array}$ & $\begin{array}{c}\text { PR patients } \\
(n=511)\end{array}$ & $\begin{array}{c}\text { P } \\
\text { value }\end{array}$ \\
\hline Any associated AID & $19.4 \%(n=28)$ & $4.7 \%(n=24)$ & $<0,001$ \\
\hline Rheumatoid Arhritis & $11.1 \%(n=16)$ & - & - \\
\hline $\begin{array}{l}\text { Systemic Lupus } \\
\text { Erythematosus }\end{array}$ & $5.6 \%(n=8)$ & $0.4 \%(n=2)$ & $<0.001$ \\
\hline Autoimmune Thyroidis & $4.9 \%(n=7)$ & $1.8 \%(n=9)$ & 0.059 \\
\hline Sjögren's Syndrome & $4.2 \%(n=6)$ & $2.3 \%(n=12)$ & NS \\
\hline $\begin{array}{l}\text { Dermatomyositis/ } \\
\text { polymyositis }\end{array}$ & $2.1 \%(n=3)$ & 0 & 0.01 \\
\hline $\begin{array}{l}\text { Primary Biliary } \\
\text { Cirrhosis }\end{array}$ & $0.7 \%(n=1)$ & 0 & NS \\
\hline $\begin{array}{l}\text { Type 1 Diabetes } \\
\text { mellitus }\end{array}$ & $0.7 \%(n=1)$ & 0 & NS \\
\hline $\begin{array}{l}\text { Antiphospholipid } \\
\text { Syndrome }\end{array}$ & $0.7 \%(n=1)$ & $0.2 \%(n=1)$ & NS \\
\hline
\end{tabular}


registered $6.9 \%(\mathrm{n}=10) \mathrm{SSc}$ patients associating two AIDs, and 2 SSc patients presented more than two AIDs (3 and 4 AIDs, respectively). The most prevalent associations with SSc were SLE + SS, RA + AIT, or RA + SLE. There was only one patient in the RA cohort associating 2 other AIDs.

\section{Characteristics of patients with SSc and AID}

There were no significant differences regarding the demographic data between patients with SSc associating AID and patients with RA associating AID (Table 3). Patients with lcSSc tended to associate more frequently other AIDs than patients with diffuse cutaneous SSc (dcSSc).

TABLE 3. Demographic characteristics of patients with $\mathrm{SSC}$ with and without associated autoimmune disease (AID)

\begin{tabular}{|l|c|c|c|}
\hline & $\begin{array}{c}\text { SSc+AID } \\
(\mathbf{n}=\mathbf{2 8})\end{array}$ & $\begin{array}{c}\text { SSc, } \text { no other } \\
\text { AID }(\mathbf{n}=144)\end{array}$ & $\begin{array}{c}\mathbf{p} \\
\text { value }\end{array}$ \\
\hline $\begin{array}{l}\text { Age at inclusion, years } \\
\text { (mean } \pm \text { SD) }\end{array}$ & $56.8 \pm 10.5$ & $54.3 \pm 12.3$ & NS \\
\hline $\begin{array}{l}\text { Age at diagnosis, years } \\
\text { (mean } \pm \text { SD) }\end{array}$ & $49.58 \pm 12.9$ & $47.76 \pm 12.91$ & NS \\
\hline $\begin{array}{l}\text { Disease duration, years } \\
\text { (mean } \pm \text { SD) }\end{array}$ & $7.2 \pm 6.1$ & $6.5 \pm 6.4$ & NS \\
\hline Female gender & $100 \%$ & $88.9 \%$ & NS \\
\hline $\begin{array}{l}\text { IcSSc } \\
\text { Survival rate } \\
\text { Initial symptom - }\end{array}$ & $85.71 \%$ & $48.7 \%$ & 0,001 \\
Raynaud's Phenomenon & $92.8 \%$ & $89.7 \%$ & NS \\
\hline
\end{tabular}

As expected, SSc patients with other AIDs presented more frequently musculoskeletal involvement and sicca symptoms in comparison with SSc patients without AIDs: arthritis (29.6\% vs. $18.3 \%$, $\mathrm{p}=0.18)$, arthralgiae $(71.4 \%$ vs. $59.5 \%, \mathrm{p}=0.24)$, muscle weakness $(32.1 \%$ vs. $26.5 \%, \mathrm{p}=0.55)$, xerostomy $(32.0 \%$ vs. $26.9 \%, \mathrm{p}=0.6)$, xerophtalmia ( $36 \%$ vs. $11 \%, p=0.005)$, however except for xerophtalmia these did not reach statistical significance.

\section{Outcomes of SSc patients with AIDs}

Despite the fact that we did not obtain statistically significant correlations, we observed a trend to a better outcome regarding the cardiac, vascular and pulmonary involvement in patients with SSc and associated AIDs. We also recorded less deaths in patients with SSc and AIDs (7.1\% vs. 10.3\%). Clinical parameters associated with a poor outcome like calcinosis, tendon friction rubs, hipo/hyperpigmentation and telangiectasiae are analyzed in Table 4.
TABLE 4. Outcomes of patients with SSc and associated AIDs, compared to patients who have only SSC

\begin{tabular}{|l|c|c|}
\hline & $\begin{array}{c}\text { SSc with AIDs } \\
(\mathbf{n}=\mathbf{2 8})\end{array}$ & $\begin{array}{c}\text { SSc } \\
(\mathbf{n}=116)\end{array}$ \\
\hline Deceased patients & $\mathbf{7 . 1 \%}$ & $10.3 \%$ \\
\hline Palpitation & $\mathbf{2 5 \%}$ & $\mathbf{5 2 . 2 \%}$ \\
\hline Cardiac conduction abnormalities & $\mathbf{1 1 . 1 \%}$ & $\mathbf{2 1 . 2 \%}$ \\
\hline Arrythmia & $\mathbf{7 . 1 \%}$ & $\mathbf{9 . 6 \%}$ \\
\hline Forced Vital Capacity $<80 \%$ & $13 \%$ & $\mathbf{3 0 . 9 \%}$ \\
\hline $\begin{array}{l}\text { Diffusing capacity of the lung for } \\
\text { carbon monoxide }<75 \%\end{array}$ & $69.6 \%$ & $\mathbf{7 8 . 7 \%}$ \\
\hline Digital Ulcers & $10.7 \%$ & $\mathbf{2 6 . 7 \%}$ \\
\hline Digital pitting scars & $\mathbf{4 2 . 9 \%}$ & $\mathbf{5 4 . 3 \%}$ \\
\hline Tendon friction rubs & $\mathbf{0 \%}$ & $\mathbf{1 1 . 3 \%}$ \\
\hline Calcinosis & $\mathbf{2 1 . 4 \%}$ & $\mathbf{2 7 . 2 \%}$ \\
\hline Telangiectasiae & $\mathbf{5 9 . 3 \%}$ & $\mathbf{6 6 . 1 \%}$ \\
\hline Hipo/Hyperpigmentation & $\mathbf{5 0 \%}$ & $\mathbf{6 0 \%}$ \\
\hline
\end{tabular}

\section{Skin involvement in SSc patients with AIDs}

Dividing the SSc patients without AIDs in the two LeRoy disease subsets (dcSSc and $1 \mathrm{cSSc}$ ) and comparing the mRSS with the SSc patients with AIDs we noticed that in SSc + AIDs patients, as well as in patients with $1 \mathrm{cSSc}$, mRSS remains rather stable during the disease course, while in dcSSc the value of mRSS decreases in time. The mean value of the yearly mRSS was lower in the patients SSc with AIDs (Figure 1).

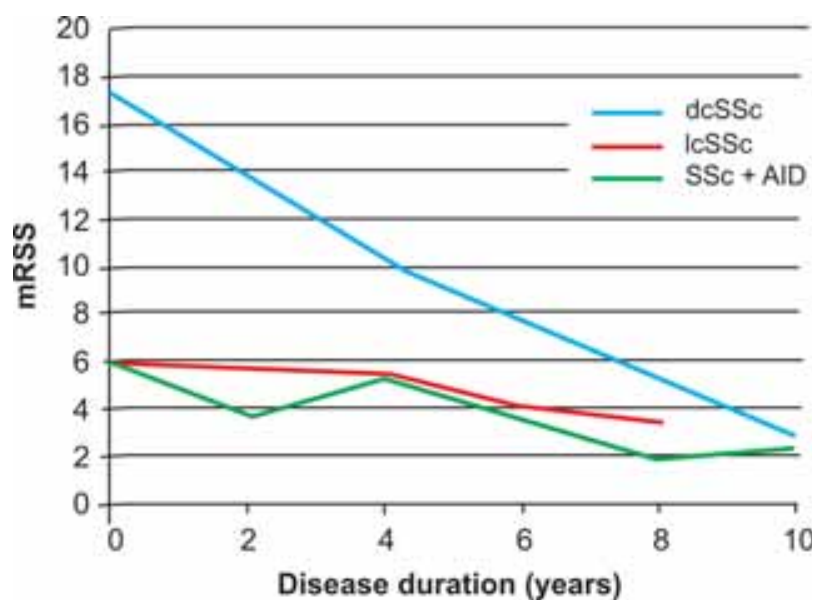

FIGURE 1. Evolution of mean subgroup mRSS during the follow up of patients with SSc and AIDs in comparison with patients with (only) IcSSc or dcSSc

\section{DISCUSSION}

We have shown in our study that patients with SSc can associate more frequently other AIDs, not only in comparison to general population, but also to patients with RA. The exact prevalence of the associated AIDs is still a matter of debate. Different studies show a varying prevalence of AID from $11 \%$ to 
$40 \%$ (5). A recent meta-analysis concluded that the prevalence of AID in SSc patients is 26\% (6), with AIT being the most prevalent $(10,4 \%)$, followed by SS $(7,7 \%)$ and DM/PM (5,6\%). The prevalence of AID in our cohort was lower, rising the suspicion of underdiagnosed patients. Comparing the prevalence of AIDs in SSc patients with the one in RA control cohort and in the general population (3-6\%) (7) we found that frequency of AIDs is almost 4 times higher in SSc patients. Our study assigns RA as the most frequent association in SSc patients. We do not exclude the possibility of an overestimated number of RA patients due to severity of the musculoskeletal involvement that sometimes is difficult to classify. When applying the 2010 Criteria for Rheumatoid Arthritis on the SSc patients they were satisfied by less than $50 \%$ of patients. But the new classification criteria for RA are designed for identifying the subset of patients who are presenting with an unexplained inflammatory arthritis of a peripheral joint, so they should not be used in patients with other well established conditions (8). Patients with SSc should be regarded as a special subset with particular diagnostic and therapeutic approach.

Dealing with a heterogeneous disease as SSc, it is sometimes difficult cu distinguish the manifestations of the disease from an overlapping syndrome. In this regard, in the last years, many researchers have approached this field and discovered antibodies that can provide a better classification of SSc patients (9). In our study we bring evidence that dividing SSc patients not only in two groups (lcSSc and dcSSc) but also in a third one consisting of SSc patients with AIDs provides more information about their outcome, leading to a better tailored management. This is of major importance especially in a disease with

\section{REFERENCES}

1. Elhai M., et al. Trends in mortality in patients with systemic sclerosis over 40 years: a systematic review and meta-analysis of cohort studies. Rheumatology (Oxford), 2012. 51(6): p. 1017-26.

2. Allanore Y. , Dieude P. and Boileau C. Genetic background of systemic sclerosis: autoimmune genes take centre stage. Rheumatology (Oxford), 2010. 49(2): p. 203-10.

3. Rodriguez-Reyna T.S., Alarcon-Segovia D. Overlap syndromes in the context of shared autoimmunity. Autoimmunity, 2005. 38(3): p. 219-23.

4. Rose N.R., Bona C. Defining criteria for autoimmune diseases (Witebsky's postulates revisited). Immunol Today, 1993. 14(9): p. 426-30.

5. Avouac J., et al. Associated autoimmune diseases in systemic sclerosis define a subset of patients with milder disease: results from 2 large cohorts of European Caucasian patients. J Rheumatol, 2010. 37(3): p. 608-14. such an unpredictable course as SSc. We have shown that SSc patients with AIDs tend to have a better outcome. Our study included a relatively small number of patients, which may account for not reaching statistical significance in the comparison regarding disease outcome. Even though, our data showed the same trend regarding the prognosis of SSc patients with AIDs as in an extended study on SSc-overlap patients, providing evidence that overlap patients have a better prognosis than patients with dcSSc but not as good as patients with lcSSc (10). Moreover, we included in our analysis also patients with other AIDs (not only overlap syndromes), but unfortunately we could not compare their outcome with lcSSc and dcSSc as separate subsets because of the small number of patients. Finally, other limitations of our study were loss to follow up of patients and missing data due to the limited access to immunological testing.

\section{CONCLUSION}

In conclusion, associated AIDs are rather frequent in SSc, affecting almost $20 \%$ of the patients. Taking in consideration the better outcome of patients with SSc and associated AIDs, they could be regarded as a separate subset of SSc.

\section{Acknowledgement}

This paper was co-financed from the European Social Fund, through the Sectorial Operational Program Human Resources Development 2007-2013, project number POSDRU/159/1.5/S/138907 „Excellence in scientific interdisciplinary research, doctoral and postdoctoral, in the economic, social and medical fields - EXCELIS“, coordinator The Bucharest University of Economic Studies.

6. Elhai M., et al. Systemic sclerosis at the crossroad of polyautoimmunity. Autoimmun Rev, 2013. 12(11): p. 1052-7.

7. Cardenas-Roldan J., Rojas-Villarraga A., Anaya J.M. How do autoimmune diseases cluster in families? A systematic review and meta-analysis. BMC Med, 2013. 11: p.73.

8. Aletaha D., et al. 2010 Rheumatoid arthritis classification criteria: an American College of Rheumatology/European League Against Rheumatism collaborative initiative. Arthritis Rheum, 2010. 62(9): p. 2569-81.

9. Mehra S., et al. Autoantibodies in systemic sclerosis. Autoimmun Rev, 2013. 12(3): p. 340-54.

10. Moinzadeh P., et al. Disease progression in systemic sclerosisoverlap syndrome is significantly different from limited and diffuse cutaneous systemic sclerosis. Ann Rheum Dis, 2015. 74(4): p. $730-7$. 